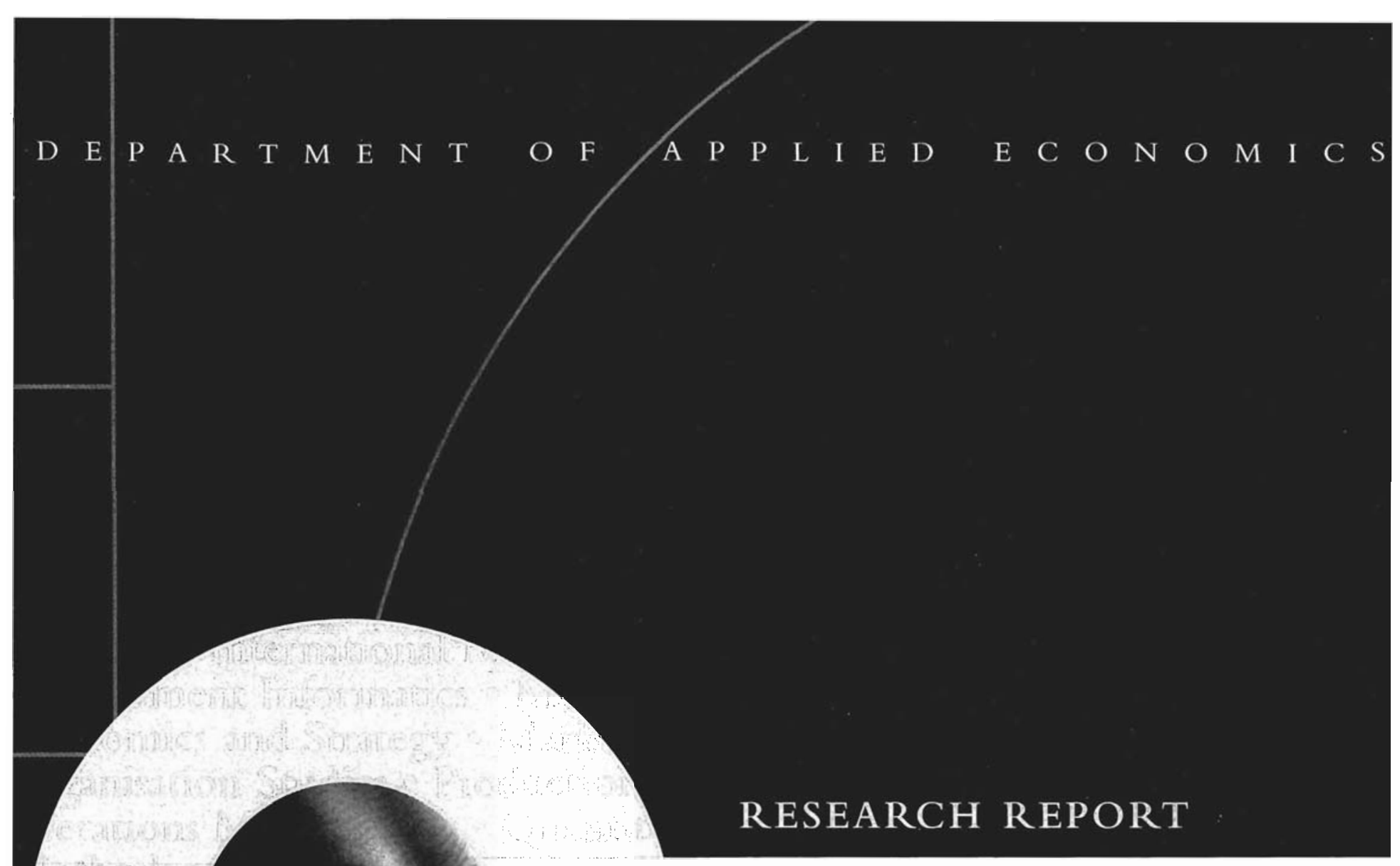

APPROXIMATION ALGORITHMS FOR RECTANGLE STABBING AND INTERVAL STABBING PROBLEMS SOFia KovaleVa • Frits C.R. SPIEKSMA

OR 0433 


\title{
Approximation Algorithms for Rectangle Stabbing and Interval Stabbing Problems*
}

\author{
Sofia Kovaleva ${ }^{\dagger}$ and Frits C.R. Spieksma ${ }^{\ddagger}$
}

\begin{abstract}
In the weighted rectangle stabbing problem we are given a grid in $\mathbb{R}^{2}$ consisting of columns and rows each having a positive integral weight, and a set of closed axis-parallel rectangles each having a positive integral demand. The rectangles are placed arbitrarily in the grid with the only assumption that each rectangle is intersected by at least one column and at least one row. The objective is to find a minimum-weight (multi)set of columns and rows of the grid so that for each rectangle the total multiplicity of selected columns and rows stabbing it is at least its demand. A special case of this problem arises when each rectangle is intersected by exactly one row. We describe two algorithms, called STAB and ROUND, that are shown to be constant-factor approximation algorithms for different variants of this stabbing problem.
\end{abstract}

\section{Introduction.}

The weighted rectangle stabbing problem (WRSP) can be described as follows: given is a grid in $\mathbb{R}^{2}$ consisting of columns and rows each having a positive integral weight, and a set of closed axis-parallel rectangles each having a

${ }^{*}$ This work grew out of the Ph.D. thesis [5]. This research was supported by EU-grant APPOL, IST 2001-30027.

${ }^{\dagger}$ Corresponding author, Department of Quantitative Economics, Maastricht University, P.O. Box 616, NL-6200 MD Maastricht, The Netherlands, e-mail: sonja.kovaleva@ke.unimaas.nl.

‡Department of Applied Economics, Katholieke Universiteit Leuven, Naamsestraat 69, B-3000, Leuven, Belgium, e-mail: frits.spieksma@econ.kuleuven.ac.be. 
positive integral demand. The rectangles are placed arbitrarily in the grid with the only assumption that each rectangle is intersected by at least one column and at least one row. The objective is to find a minimum-weight (multi)set of columns and rows of the grid so that for each rectangle the total multiplicity of selected columns and rows stabbing this rectangle equals at least its demand. (A column or row is said to stab a rectangle if it intersects it.)

A special case of the WRSP is the case where each rectangle is intersected by exactly one row; we will refer to the resulting problem as the weighted interval stabbing problem (WISP), or ISP in case of unit weights (see Figure 1 for an example of an instance of the ISP).

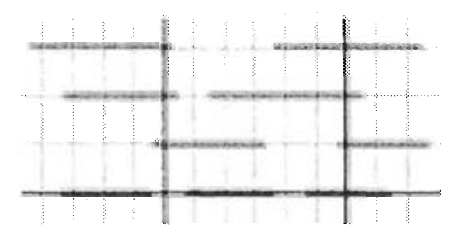

Figure 1: An instance of ISP with unit demands. The rectangles (or intervals in this case) are in grey; the columns and row in black constitute a feasible solution.

Motivation. Although at first sight the WRSP may seem rather specific, it is not difficult to see that the following two problems can be reduced to WRSP:

- Solving special integer programming problems: the following type of integer linear programming problems can be reformulated as instances of WRSP: minimize $\left\{w x \mid(B \mid C) x \geq b, x \in \mathbb{Z}^{l}\right\}$, where $B$ and $C$ are both 0,1-matrices with consecutive '1'-s in the rows (a so-called interval matrix, see e.g. Schrijver [8]), $b \in \mathbb{Z}_{+}^{n}, w \in \mathbb{Z}_{+}^{l}$. Indeed, construct a grid which has a column for each column in $B$ and a row for each column in $C$. For each row $i$ of matrix $B \mid C$, draw a rectangle $i$ such that it intersects only the columns and rows of the grid corresponding to the positions of ' 1 '-s in row $i$. Observe that this construction is possible since $B$ and $C$ have consecutive '1'-s in the rows. To complete the construction, assign demand $b_{i}$ to each rectangle $i$ and a corresponding weight $w_{j}$ to each column and row of the grid. Let the decision variables $x$ describe the multiplicities of the columns and rows of the grid. In this 
way we have obtained an instance of WRSP. In other words, integer programming problems where the columns of the constraint matrix $A$ can be permuted such that $A=(B \mid C)$ with $B$ and $C$ each being an interval matrix, is a special case of WRSP.

- Stabbing geometric figures in the plane: given a set of arbitrary connected closed geometric sets in the plane, use a minimum number of straight lines of two given directions to stab each of these sets at least once. Indeed, by introducing a new coordinate system specified by the two directions, and by replacing each closed connected set by a closed rectangle defined by the projections of the set to the new coordinate axes, we obtain an instance of the problem of stabbing rectangles using a minimum number of axis-parallel lines. More specifically, we define a grid whose rows and columns are axes-parallel lines containing the rectangles' edges. We can restrict attention to those lines since any axis-parallel line stabbing some set of rectangles can be replaced by a line stabbing this set and containing a rectangle's edge. Therefore, the problem of stabbing the rectangles with axis-parallel lines reduces to the problem of stabbing them with the rows and columns of the grid.

Literature. The WRSP and its special case WISP have received attention in literature before. Motivated by an application in parallel processing, Gaur et al. [2] present a 2-approximation algorithm for the WRSP with unit weights and demands, which admits an easy generalization to arbitrary weights and demands. Furthermore, Hassin and Megiddo [3] (mentioning military and medical applications) study a number of special cases of the problem of stabbing geometric figures in $\mathbb{R}^{2}$ by a minimum number of straight lines. In particular, they present a 2-approximation algorithm for the task of stabbing connected figures of the same shape and size with horizontal and vertical lines. Moreover, they study the case of stabbing horizontal line segments of length $K$, whose endpoints have integral $x$-coordinates, with a minimum number of horizontal and vertical lines, and give a $2-\frac{1}{K}$-approximation algorithm for this problem. In our setting this corresponds to the ISP with unit demands, where each rectangle in the input is intersected by exactly $K$ columns.

Finally, concerning computational complexity, a special case of ISP where each rectangle is stabbed by at most two columns, is shown to be APX-hard in $[7]$. 
Our results. We present two approximation algorithms for different variants of WRSP (see e.g. Vazirani [9] for an overview on approximation algorithms). First, we describe a $\frac{q+1}{q}$-approximation algorithm called ROUND for the case where the demand of each rectangle is bounded from below by an integer $q$. Observe that this provides a 2-approximation algorithm for the WRSP described in the introduction, where $q=1$. Thus, our algorithm is an improvement upon the approximation ratio of the algorithm of Gaur et al. [2] for instances with a lower bound on the rectangles' demands that is larger than 1. Second, we present a $\frac{1}{\left(1-(1-1 / k)^{k}\right)}$-approximation algorithm called $S T A B$ for $\mathrm{ISP}_{k}$, the variant of ISP where each row intersects at most $k$ rectangles (e.g., the instance depicted in Figure 1 is an instance of $\mathrm{ISP}_{3}$ ). Observe that $S T A B$ is a $\frac{4}{3}$-approximation algorithm for the case $k=2$, and that $S T A B$ is a $\frac{e}{e-1}$-approximation algorithm for the case where the number of rectangles sharing a row is unlimited $(k=\infty)$. Thus, STAB improves upon the results described in Hassin and Megiddo [3] (for $K \geq 3$ ) and does not impose any restrictions on the number of columns intersecting rectangles. Third, we state here that $S T A B$ for the weighted case of $\mathrm{ISP}_{\infty}$, i.e., the case where the columns and the rows of the grid have arbitrary positive integral weights, is a $\frac{e}{e-1}$-approximation algorithm. For the proof of this result, we refer to Kovaleva [5]. Our algorithms are based on rounding the linear programming relaxation of an integer programming formulation in an interesting way. We use the following property present in our formulation: the variables can be partitioned into two sets such that when given the values of one set of variables, one can compute in polynomial time the optimal values of the variables of the other set of variables, and vice versa. Next, we consider different ways of rounding one set of variables, and compute each time the values of the remaining variables, while keeping the best solution.

Summarizing our results:

- we generalize the results of Gaur et al. [2] to obtain a $\frac{q+1}{q}$-approximation algorithm called ROUND for the case where the demand of each rectangle is bounded from below by an integer $q$ (Section 3),

- we describe an $\frac{1}{\left(1-(1-1 / k)^{k}\right)}$-approximation algorithm called STAB for $\mathrm{ISP}_{k}$ based on an original rounding idea (Section 4).

We also show that there exist instances of the WRSP, $\mathrm{ISP}_{2}$ and $\mathrm{ISP}_{\infty}$, for which the ratio between the values of a natural ILP formulation and its LPrelaxation is equal (or arbitrary close) to the obtained approximation ratios. 
This suggests that these approximation ratios are unlikely to be improved by an LP-rounding algorithm based on the natural ILP formulation.

\section{Preliminaries.}

Let us formalize the definition of WRSP. Let the grid in the input consist of $t$ columns and $m$ rows, numbered consecutively from left to right and from bottom to top, with positive weight $w_{c}\left(v_{r}\right)$ attached to each column $c$ (row $r)$. Further, we are given $n$ rectangles such that rectangle $i$ has demand $d_{i} \in \mathbb{Z}_{+}$and is specified by leftmost column $l_{i}$, rightmost column $r_{i}$, top row $t_{i}$ and bottom row $b_{i}$.

Let us give a natural ILP formulation of WRSP. In this paper we use notation $[a: b]$ for the set of integers $\{a, a+1, \ldots, b\}$. The decision variables $y_{c}, z_{r} \in \mathbb{Z}_{+}$, $c \in[1: t], r \in[1: m]$, denote the multiplicities of column $c$ and row $r$ respectively.

$$
\begin{array}{rll}
\text { Minimize } & \sum_{c=1}^{t} w_{c} y_{c}+\sum_{r=1}^{m} v_{r} z_{r} & \\
\text { subject to } & \sum_{r \in\left[b_{i}: t_{i}\right]} z_{r}+\sum_{c \in\left[l_{i}: r_{i}\right]} y_{c} \geq d_{i} & \forall i \in[1: n] \\
z_{r}, y_{c} \in \mathbb{Z}_{+}^{1} & \forall r, c .
\end{array}
$$

The linear programming relaxation is obtained when replacing the integrality constraints (3) by the nonnegativity constraints $z_{r}, y_{c} \geq 0, \forall r, c$.

For an instance $\mathcal{I}$ of WRSP and a vector $b \in \mathbb{Z}^{n}$, we introduce two auxiliary ILP problems:

$$
\begin{array}{cccc}
\operatorname{IP}^{y}(\mathcal{I}, b): & \text { Minimize } & \sum_{c=1}^{t} w_{c} y_{c} & \\
& \text { subject to } & \sum_{c \in\left[l_{i}: r_{i}\right]} y_{c} \geq b_{i} & \forall i \in[1: n] \\
& y_{c} \in \mathbb{Z}_{+}, & \forall c \in[1: t] \\
& \text { Minimize } & \sum_{r=1}^{m} v_{r} z_{r} & \\
\operatorname{IP}^{z}(\mathcal{I}, b): & \text { subject to } & \sum_{r \in\left[b_{i}: t_{i}\right]} z_{r} \geq b_{i} & \forall i \in[1: n] \\
& & z_{r} \in \mathbb{Z}_{+}, & \forall c \in[1: m]
\end{array}
$$

Lemma 2.1. For any $b \in \mathbb{Z}^{n}$, the LP-relaxation of each of the problems $I P^{z}(\mathcal{I}, b)$ and $I P^{y}(\mathcal{I}, b)$ is integral.

Proof. This follows from the unimodularity of the constraint matrix of (5) which is implied by the "consecutive one's"-property (see e.g. Schrijver [8]). 
Corollary 2.2. The optimum value of $\operatorname{IP}^{y}(\mathcal{I}, b)\left(I P^{z}(\mathcal{I}, b)\right)$ is smaller than or equal to the value of any feasible solution to its LP-relaxation.

Corollary 2.3. The problem $I P^{y}(\mathcal{I}, b)\left(I P^{z}(\mathcal{I}, b)\right)$ can be solved in polynomial time. Its optimal solution coincides with that of its LP-relaxation.

In fact, the special structure of $\operatorname{IP}^{y}(\mathcal{I}, b)\left(\operatorname{IP}^{z}(\mathcal{I}, b)\right)$ allows us to solve it via a minimum-cost flow algorithm: let $\operatorname{MCF}(p, q)$ denote the time needed to solve the minimum cost flow problem on a network with $p$ nodes and $q$ arcs.

Lemma 2.4. The problem $I P^{y}(\mathcal{I}, b)\left(I P^{z}(\mathcal{I}, b)\right)$ can be solved in time $O(M C F(t, n+$ t)) $(O(M C F(m, n+m)))$.

Proof. Consider the LP-relaxation of formulation $\operatorname{IP}^{y}(\mathcal{I}, b)$ and substitute variables with new variables $u_{0}, \ldots, u_{t}$ as $y_{c}=u_{c}-u_{c-1}, \forall c \in[1: t]$. Then it transforms into

$$
\begin{array}{ll}
\text { Minimize } & -w_{1} u_{0}+\left(w_{1}-w_{2}\right) u_{2}+\ldots+\left(w_{t-1}-w_{t}\right) u_{t-1}+w_{t} u_{t} \\
\text { subject to } & u_{r_{i}}-u_{l_{i}-1} \geq b_{i}, \quad \forall i \in[1: n] \\
& u_{c}-u_{c-1} \geq 0, \quad \forall c \in[1: t] .
\end{array}
$$

Let us denote the vector of objective coefficients, the vector of right-hand sides and the constraint matrix by $w, b$ and $C$ respectively, and the vector of variables by $u$. Then (4) can be represented as $\{$ minimize $w u \mid C u \geq b\}$. Its dual is: $\left\{\right.$ maximize $b x \mid C^{T} x=w, x \geq 0$ \}. Observe that this is a minimum cost flow formulation with flow conservation constraints $C^{T} x=w$, since $C^{T}$ has exactly one ' 1 ' and one '-1' in each column. Given an optimal solution to the minimum cost flow problem, one can easily obtain the optimal dual solution $u_{0}, \ldots, u_{t}$ (see Ahuja et al. [1]), and thus optimal $y_{1}, \ldots, y_{t}$ as well.

\section{The Algorithm ROUND.}

Let $\mathrm{WRSP} q$ be the special case of the WRSP, where $d_{i} \geq q, \forall i \in[1: n]$. In Subsection 3.1 we describe an algorithm ROUND, and show that it achieves a ratio of $\frac{q+1}{q}$. Subsection 3.2 shows that the integrality gap between a natural integer programming formulation and its corresponding LP-relaxation equals the same ratio. 
1. solve the LP-relaxation of (1)-(3) for $\mathcal{I}$ and obtain its optimal solution $\left(y^{\mathrm{lp}}, z^{\mathrm{lp}}\right)$

2. solve $\operatorname{IP}^{y}(\mathcal{I}, a)$, where $a_{i}=\left\lfloor\frac{q+1}{q} \sum_{c \in\left[l_{i}: r_{i}\right]} y_{c}^{\text {lp }}\right\rfloor$, for all $i \in[1: n]$; obtain $\bar{y}$;

3. solve $\operatorname{IP}^{z}(\mathcal{I}, b)$, where $b_{i}=\left\lfloor\frac{q+1}{q} \sum_{c \in\left[b_{i}: t_{i}\right]} z_{c}^{\mathrm{lp}}\right\rfloor$, for all $i \in[1: n]$; obtain $\bar{z}$;

4. $\operatorname{return}(\bar{y}, \bar{z})$

Figure 2: Algorithm ROUND .

\subsection{An approximation result.}

Figure 2 describes algorithm ROUND applied to an instance $\mathcal{I}$ of WRSPq.

Theorem 3.1. Algorithm ROUND is a $\frac{q+1}{q}$-approximation algorithm for the problem WRSPq.

Proof. Let $\mathcal{I}$ be an instance of WRSP $q$. First, we show that the solution $(\bar{y}, \bar{z})$ returned by $R O U N D$ is feasible for $\mathcal{I}$, i.e., satisfies constraints (2) and (3). Obviously, vectors $\bar{y}$ and $\bar{z}$ are integral, hence, constraint (3) is satisfied. For each $i \in[1: n]$, consider the left-hand side of constraint $(2)$ for $(\bar{y}, \bar{z})$. By construction, $\bar{y}$ and $\bar{z}$ are feasible to $\operatorname{IP}^{y}(\mathcal{I}, a)$ and $\operatorname{IP}^{z}(\mathcal{I}, b)$ respectively. Using this, and the way vectors $a$ and $b$ were constructed, obtain:

$$
\sum_{r \in\left[b_{i}: t_{i}\right]} \bar{z}_{r}+\sum_{c \in\left[l_{i}: r_{i}\right]} \bar{y}_{c} \geq a_{i}+b_{i}=\left\lfloor\frac{q+1}{q} \sum_{c \in\left[l_{i}: r_{i}\right]} y_{c}^{\mathrm{lp}}\right\rfloor+\left\lfloor\frac{q+1}{q} \sum_{r \in\left[b_{i}: t_{i}\right]} z_{r}^{\mathrm{lp}}\right\rfloor .
$$

Since $\lfloor\alpha\rfloor+\lfloor\beta\rfloor \geq\lfloor\alpha+\beta\rfloor-1$, for any positive $\alpha$ and $\beta$, and since $z^{\text {lp }}$ and $y^{\text {lp }}$ satisfy constraint $(2)$, the right-hand side of $(7)$ is at least equal to:

$$
\left\lfloor\frac{q+1}{q}\left(\sum_{c \in\left[l_{i}: r_{i}\right]} y_{c}^{\mathrm{lp}}+\sum_{r \in\left[b_{i}: t_{i}\right]} z_{r}^{\mathrm{lp}}\right)\right\rfloor-1 \geq\left\lfloor\frac{q+1}{q} d_{i}\right\rfloor-1 \geq d_{i}+1-1=d_{i},
$$

where the last inequality holds because $d_{i} \geq q, \forall i \in[1: n]$ for WRSP $q$. Thus, inequality $(2)$ holds for $(\bar{y}, \bar{z})$ and hence $(\bar{y}, \bar{z})$ constitutes a feasible solution to instance $\mathcal{I}$ of $\mathrm{WRSP} q$. 
The approximation ratio of ROUND is obtained from the ratio between the value of the returned solution $(\bar{y}, \bar{z})$ and the value of the optimal fractional solution $\left(y^{\mathrm{lp}}, z^{\mathrm{lp}}\right)$ (and of course by observing that the value of the latter solution does not exceed the optimum value of $\mathrm{WRSP} q$ ).

We claim that $\sum_{c=1}^{t} w_{c} \bar{y}_{c} \leq \frac{q+1}{q} \sum_{c=1}^{t} w_{c} y_{c}^{\mathrm{lp}}$. Observe that $\frac{q+1}{q} y^{\mathrm{lp}}$ is a feasible solution to the LP-relaxation of $\operatorname{IP}^{y}(\mathcal{I}, a)$ with $a_{i}=\left\lfloor\frac{q+1}{q} \sum_{c \in\left[l_{i}: r_{i}\right]} y_{c}^{\text {lp }}\right\rfloor, \forall i \in$ $\left[1:{ }_{1}: n\right]$. Indeed, constraints (4) are clearly satisfied: $\sum_{c \in\left[l_{i}: r_{i}\right]} \frac{q+1}{q} y_{c}^{\mathrm{lp}} \geq$ $\left\lfloor\frac{q+1}{q} \sum_{c \in\left[l_{i}: r_{i}\right]} y_{c}^{\mathrm{lp}}\right\rfloor, \forall i \in[1: n]$. Thus, vectors $\bar{y}$ and $\frac{q+1}{q} y^{\mathrm{lp}}$ are respectively an optimal solution to $\operatorname{IP}^{y}(\mathcal{I}, a)$ and a feasible solution to its LP-relaxation. Now Corollary 2.2 implies the claim.

Similarly, $\sum_{r=1}^{m} v_{r} \bar{z}_{r} \leq \frac{q+1}{q} \sum_{r=1}^{m} v_{r} z_{r}^{\mathrm{lp}}$. This proves the ratio of $\frac{q+1}{q}$ between the value of solution $(\bar{y}, \bar{z})$ and solution $\left(y^{\mathrm{lp}}, z^{\mathrm{lp}}\right)$.

Observe that in case of unit weights and unit demands, ROUND boils down to the algorithm described in Gaur et al. [2].

\subsection{Tightness.}

In this section we provide instances showing that the ratio between the the optimal value of WRSP $q$ and the LP-relaxation of its natural ILP formulation (1)-(3) can be arbitrary close to $\frac{q+1}{q}$, the approximation factor of algorithm ROUND. We will refer to this ratio as the integrality gap of (1)-(3).

Theorem 3.2. For each $q \in \mathbb{N}$, the integrality gap of (1)-(3) is arbitrarily close to $\frac{q+1}{q}$.

Proof. For any $q \in \mathbb{N}$ and any integral $n \geq 2$, we construct an instance $\mathcal{I}_{n}^{q}$ of WRSP $q$, such that the ratio between the optimal values of the formulation (1)-(3) and its LP-relaxation for the instance $\mathcal{I}_{n}^{q}$ tends to $\frac{q+1}{q}$ as $n$ increases. The construction is as follows. Denote: $\varepsilon=\frac{1}{2(n-1)^{2}(q+1)^{2}}$, and let the grid consist of $q n / \varepsilon$ columns and $q n / \varepsilon$ rows. The instance includes all different rectangles intersecting exactly $q / \varepsilon$ columns and rows in total (here 'different' implies intersecting different subsets of columns and rows). The weight of each column and row is unit, and the demand of each rectangle is equal to $q$.

Claim 1. The optimum value of the LP-relaxation of formulation (1)-(3) for $\mathcal{I}_{n}^{q}$ is less than or equal to $2 q n$.

To show this, we introduce a feasible solution to the LP relaxation for $\mathcal{I}_{n}^{q}$ of the value $2 q n$ : assign multiplicity $\varepsilon$ to each column and row of the grid. It 
is feasible, since each rectangle in $\mathcal{I}_{n}^{q}$ is then stabbed by a total multiplicity of $q$. The value of this solution is $2 q n$, hence the claim follows.

Claim 2. The optimum value of $\mathrm{WRSP} q$ for $\mathcal{I}_{n}^{q}$ is greater than $2(n-1)(q+1)$. Suppose the opposite is true, that is, suppose that there exists a feasible (integral) solution to $\mathrm{WRSP} q$ for $\mathcal{I}_{n}^{q},(y, z)$, with value $2(n-1)(q+1)$. Suppose that this solution assigns total multiplicity $C$ to the columns and total multiplicity $R$ to the rows of the grid $\left(\sum_{c=1}^{n q / \varepsilon} y_{c}=C, \sum_{r=1}^{n q / \varepsilon} z_{r}=R\right.$, $C+R=2(n-1)(q+1))$. Denote by $x$ the maximum number of consecutive columns having total multiplicity less than or equal to $q-1$.

Claim 2.1. $x \geq \frac{n q / \varepsilon}{C / q+1}-1$.

Indeed, by definition of $x$, the total multiplicity of any $x+1$ consecutive columns is at least $q$. Therefore, the total multiplicity $C$ of the columns has to be at least $\left\lfloor\frac{n q / \varepsilon}{x+1}\right\rfloor \cdot q$. This inequality, i.e., $C \geq\left\lfloor\frac{n q / \varepsilon}{x+1}\right\rfloor \cdot q$, implies the claim. Claim 2.2. $x<q / \varepsilon-1$.

Suppose that this does not hold. Since the number of rows in the grid is obviously larger than their total multiplicity $R$ (otherwise the value of our solution would be much larger than $2(n-1)(q+1))$, there exists a row with multiplicity 0 . By construction our instance contains a rectangle intersecting this row and $q / \varepsilon-1$ of the $x$ consecutive columns. Obviously, the total multiplicity of the columns and rows stabbing this rectangle is at most $q-1$, which is a contradiction with the assumption that solution $(y, z)$ is feasible.

Claim 2.3. $C>(n-1) q$. This follows from $\frac{n q / \varepsilon}{C / q+1}<q / \varepsilon$, which in turn follows from Claims 2.1 and 2.2 .

We continue with the proof of Claim 2. Consider all the rectangles in $\mathcal{I}_{n}^{q}$ intersecting exactly those $x$ consecutive columns. Each of them intersects $q / \varepsilon-x$ rows. It receives multiplicity of at most $q-1$ from the columns, and therefore needs to receive multiplicity of at least 1 from its rows. The fact that our instance contains all the possible different rectangles implies that each set of $q / \varepsilon-x$ consecutive rows has to have the total multiplicity of at least 1. By a similar argument as in the proof of Claim 2.1, and by Claim 2.1 itself, the total multiplicity of the rows has to satisfy:

$$
\begin{gathered}
R \geq\left\lfloor\frac{n q / \varepsilon}{q / \varepsilon-x}\right\rfloor \geq\left\lfloor\frac{n q / \varepsilon}{q / \varepsilon-\frac{n q / \varepsilon}{C / q+1}+1}\right\rfloor=\left\lfloor\frac{n}{1-\frac{n}{C / q+1}+\frac{\varepsilon}{q}}\right\rfloor> \\
>\frac{n}{1-\frac{n}{C / q+1}+\frac{\varepsilon}{q}}-1=\frac{n(C+q)}{C+q-n q+\frac{\varepsilon(C+q)}{q}}-1 .
\end{gathered}
$$


Multiplying both sides by the denominator, which is positive due to Claim 2.3, obtain: $R\left(C+q-n q+\frac{\varepsilon(C+q)}{q}\right)>n(C+q)-\left(C+q-n q+\frac{\varepsilon(C+q)}{q}\right)$.

Interchanging the role of columns and rows, and using a similar argument, we have: $C\left(R+q-n q+\frac{\varepsilon(R+q)}{q}\right)>n(R+q)-\left(R+q-n q+\frac{\varepsilon(R+q)}{q}\right)$. Summing up these inequalities, and collecting the coefficients of the terms $C R$ and $(C+R)$, we arrive at:

$$
\begin{gathered}
2 C R(1+\varepsilon / q)>(C+R)(-q+n q-\varepsilon+n-1-\varepsilon / q)+4 n q-2 q-2 \varepsilon= \\
\quad=(n-1)(q+1)(C+R)-(\varepsilon+\varepsilon / q)(C+R)+4 n q-2 q-2 \varepsilon .
\end{gathered}
$$

Using our assumption $C+R=2(n-1)(q+1)$, we rewrite as follows:

$2 C R(1+\varepsilon / q)>2(n-1)^{2}(q+1)^{2}-\varepsilon(1+1 / q) \cdot 2(n-1)(q+1)+4 n q-2 q-2 \varepsilon$.

Obviously, the value of the term $C R$, given that $C+R=2(n-1)(q+1)$, can not exceed $(n-1)^{2}(q+1)^{2}$. Then, if solution $(y, z)$ is feasible, the following should be satisfied (recall that $\varepsilon=\frac{1}{2(n-1)^{2}(q+1)^{2}}$ ):

$$
\begin{gathered}
2(n-1)^{2}(q+1)^{2}+\frac{1}{q}>2(n-1)^{2}(q+1)^{2}-\frac{1+1 / q}{(n-1)(q+1)}+4 n q-2 q-2 \varepsilon, \text { or, } \\
\frac{1}{q}>-\frac{1}{(n-1)(q+1)}+4 n q-2 q-\frac{1}{(n-1)^{2}(q+1)^{2}} .
\end{gathered}
$$

This inequality does not hold for any $q \geq 1, n \geq 2$. Thus, solution $(y, z)$ can not be feasible. This proves Claim 2 .

From Claims 1 and 2 it follows that the ratio between the optimum value of formulation (1)-(3) and the optimum value of its LP-relaxation for $\mathcal{I}_{n}^{q}$ is at least equal to $\frac{2(n-1)(q+1)}{2 n q}$, which tends to $\frac{q+1}{q}$ as $n$ increases.

Remark 3.1. Notice that this example shows that the results in Gaur et al. [2] are tight as well.

As mentioned in the introduction, Theorems 3.1 and 3.2 imply that it is unlikely that a better ratio for $\mathrm{WRSP} q$ can be achieved using formulation (1)-(3). 


\section{Algorithm STAB.}

Recall that the interval stabbing problem WISP $_{k}$ refers to the restriction of WRSP where each rectangle in the input is intersected by exactly one row and each row intersects at most $k$ rectangles. Moreover, we assume in this section that all the weights and demands are unit: $w_{c}=v_{r}=d_{i}=1, \forall c \in$ $[1: t], r \in[1: m]$ and $i \in[1: n]$, i.e., we concentrate on $\operatorname{ISP}_{k}$ with unit demands. In Subsection 4.1 we describe an algorithm STAB, and show that it achieves a ratio of $\frac{1}{1-(1-1 / k)^{k}}$. Subsection 4.2 shows that the integrality gap between the values of a natural integer programming formulation and its corresponding LP-relaxation equals the same approximation ratio for $k=2$ and $k=\infty$, namely $\frac{4}{3}$ and $\frac{e}{e-1}$ respectively. An alternative algorithm for the case $k=2$ yielding the same worst-case ratio (i.e., $\frac{4}{3}$ ) is described in Kovaleva and Spieksma [6].

\subsection{An approximation result.}

In this subsection we describe an algorithm $S T A B$ for $\operatorname{ISP}_{k}$ and show that it is a $\frac{1}{1-(1-1 / k)^{k}}$-approximation algorithm. Let us first adapt the ILP formulation (1)-(3) to $\operatorname{ISP}_{k}$ with unit demands:

$$
\begin{array}{ccl}
\text { Minimize } & \sum_{c=1}^{t} y_{c}+\sum_{r=1}^{m} z_{r} & \\
\text { subject to } & z_{\rho_{i}}+\sum_{c \in\left[l_{i}: r_{i}\right]} y_{c} \geq 1 & \forall i \in[1: n] \\
& z_{r}, y_{c} \in \mathbb{Z}_{+} & \forall r, c .
\end{array}
$$

Informally, algorithm STAB can be described as follows: solve the LPrelaxation of (8)-(10), and denote the solution found by $\left(y^{\mathrm{lp}}, z^{\mathrm{lp}}\right)$. Assume, without loss of generality, that the rows are sorted as $z_{1}^{\mathrm{lp}} \geq z_{2}^{\mathrm{lp}} \geq \ldots \geq z_{m}^{\mathrm{lp}}$. At each iteration $j(j=0, \ldots, m)$ we solve the problem (8)-(10) with a fixed vector $z$, the first $j$ elements of which are set to 1 , and the others to 0 . As shown in Section 2, this can be done in polynomial time using a minimum cost flow algorithm. Finally, we take the best of the resulting $m+1$ solutions. A formal description of $S T A B$ is shown in Figure 3.

We use notation: $\operatorname{value}(y, z) \equiv \sum_{c=1}^{t} y_{c}+\sum_{r=1}^{m} z_{r}$, value $(y) \equiv \sum_{c=1}^{t} y_{c}$, and $\operatorname{value}(z) \equiv \sum_{r=1}^{m} z_{r}$.

Theorem 4.1. Algorithm STAB is a $\frac{1}{1-(1-1 / k)^{k}}$-approximation algorithm for $I S P_{k}$. 
1. solve the LP-relaxation of (8)-(10), and obtain its optimal solution $\left(y^{\mathrm{lp}}, z^{\mathrm{lp}}\right)$

2. reindex the rows of the grid so that $z_{1}^{\mathrm{lp}} \geq z_{2}^{\mathrm{lp}} \geq \ldots \geq z_{m}^{\mathrm{lp}}$;

3. $V \leftarrow \infty$

4. for $j=0$ to $m$

for $i=1$ to $j \quad \bar{z}_{i} \leftarrow 1$,

for $i=j+1$ to $m \quad \bar{z}_{i} \leftarrow 0$.

solve $\operatorname{IP}^{y}(\mathcal{I}, b)$, where $b_{i}=1-\bar{z}_{\rho_{i}}, \forall i \in[1: n]$, and obtain $\bar{y}$;

if value $(\bar{y}, \bar{z})<V$ then $V \leftarrow \operatorname{value}(\bar{y}, \bar{z}), \quad y^{*} \leftarrow \bar{y}, z^{*} \leftarrow \bar{z}$;

5. $\operatorname{return}\left(y^{*}, z^{*}\right)$.

Figure 3: Algorithm STAB

Proof. Consider an instance $\mathcal{I}$ of $\operatorname{ISP}_{k}$, and let $\left(y^{\mathrm{lp}}, z^{\mathrm{lp}}\right)$ and $\left(y^{*}, z^{*}\right)$ be respectively an optimal LP solution and the solution returned by the algorithm for $\mathcal{I}$. We prove the theorem by establishing that

$$
\operatorname{value}\left(y^{*}, z^{*}\right) \leq \frac{1}{1-(1-1 / k)^{k}} \operatorname{value}\left(y^{\mathrm{lp}}, z^{\mathrm{lp}}\right) .
$$

It is enough to prove the result for instances satisfying the following assumption: we assume that the optimal LP solution satisfies constraints (9) at equality, i.e.

$$
z_{\rho_{i}}^{\mathrm{lp}}+\sum_{c \in\left(l_{i}: r_{i}\right)} y_{c}^{\mathrm{lp}}=1, \forall i \in[1: n] .
$$

Indeed, if (12) does not hold for some intervals $i$, we change the instance by shortening the appropriate intervals (and perhaps splitting the columns with $y^{\mathrm{lp}}$-values) so that the assumption becomes true (see Figure 4). It is easy to check that the optimal LP solution remains the same (up to the splitted columns). Since in the new instance the intervals become shorter, algorithm $S T A B$ returns a solution with a value equal to or larger than the value of the solution returned for the initial instance. Then inequality (11) proven for the new instance implies this inequality for the initial instance as well.

We order the rows of the grid in order of nonincreasing $z^{\mathrm{lp}}$-values, and we denote by $l(l \geq 0)$ the number of $z^{\text {pp }}$-values equal to 1 . Then: $z_{1}^{1 \mathrm{p}}=\ldots=$ 

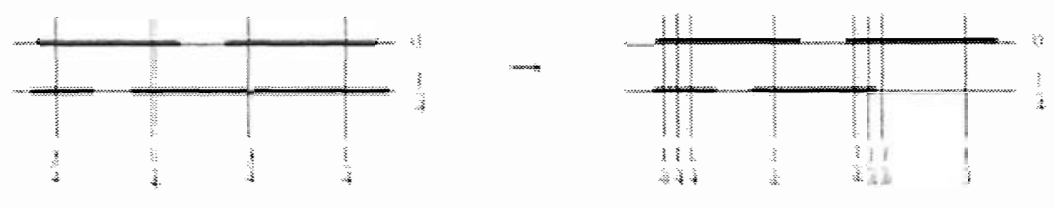

Figure 4: Example of an initial instance (left) and a new instance satisfying the assumption (right).

$z_{l}^{\mathrm{lp}}=1,1>z_{l+1}^{\mathrm{lp}} \geq \ldots \geq z_{m}^{\mathrm{lp}} \geq 0$. We assume that value $\left(y^{\mathrm{lp}}\right)$ is positive (otherwise all the $z^{\mathrm{lp}}$-values have to be equal to 1 and the theorem obviously holds).

By construction:

$$
\operatorname{value}\left(y^{*}, z^{*}\right)=\min _{j \in[0: m]} \operatorname{value}\left(y^{j}, z^{j}\right) \leq \min _{j \in[l: m]} \operatorname{value}\left(y^{j}, z^{j}\right),
$$

where $\left(y^{j}, z^{j}\right)$ is the $j$ th solution generated in Step 4 of STAB.

Let us proceed by defining for each $j \in[0: m]$, a number $q_{j} \in \mathbb{R}$ that depends on some given $\Delta \in[0,1]^{m}$ and given $\beta>0$ as follows:

$$
\left(1-\Delta_{j+1}\right)+\left(1-\Delta_{j+2}\right)+\ldots+\left(1-\Delta_{j+\left\lfloor q_{j}\right\rfloor}\right)+\left(q_{j}-\left\lfloor q_{j}\right\rfloor\right)\left(1-\Delta_{j+\left\lfloor q_{j}\right\rfloor+1}\right)=\beta
$$

where we put $\Delta_{j}=0$ if $j>m$.

Observe that $q_{j}$ for each $j \in[0: m]$ is uniquely defined by this equality; we denote the solution of $(14)$ by $q_{j}(\Delta, \beta)$.

We will prove the following lemma:

\section{Lemma 4.2.}

$$
\operatorname{value}\left(y^{j}, z^{j}\right) \leq j+k \cdot q_{j}\left(z^{l p}, \frac{\operatorname{value}\left(y^{l p}\right)}{k}\right), \forall j \in[l: m] .
$$

Then, assuming that Lemma 4.2 holds, it follows from (13) that:

$$
\operatorname{value}\left(y^{*}, z^{*}\right) \leq \min _{j \in[l: m]}\left(j+k \cdot q_{j}\left(z^{\mathrm{lp}}, \frac{\operatorname{value}\left(y^{\mathrm{lp}}\right)}{k}\right)\right) .
$$

The Theorem follows now from the following lemma, the proof of which can be found in the appendix: 
Lemma 4.3. Given are real numbers $1 \geq \Delta_{1} \geq \Delta_{2} \geq \ldots \geq \Delta_{m} \geq 0$, a positive real number $Y$, an integer $p \geq 2$, and an integer $l \geq 0$. Then the following holds:

$$
\min _{i \in[l: m]}\left(i+p \cdot q_{i}(\Delta, Y / p)\right) \leq \frac{1}{1-(1-1 / p)^{p}}\left(Y+\sum_{r=l+1}^{m} \Delta_{r}\right)+l
$$

By applying this Lemma with $p=k, \Delta=z^{\mathrm{lp}}$, and $Y=\operatorname{value}\left(y^{\mathrm{lp}}\right)$, the right-hand side of (15) can be bounded by:

$\frac{1}{1-(1-1 / k)^{k}}\left(\operatorname{value}\left(y^{\mathrm{lp}}\right)+\sum_{r=l+1}^{m} z_{r}^{\mathrm{lp}}\right)+l \leq \frac{1}{1-(1-1 / k)^{k}}\left(\operatorname{value}\left(y^{\mathrm{lp}}\right)+\sum_{r=l+1}^{m} z_{r}^{\mathrm{lp}}+l\right)$, and since $z_{1}^{\mathrm{lp}}=\ldots=z_{l}^{\mathrm{lp}}=1$, the right hand side of this last expression is equal to:

$$
\frac{1}{1-(1-1 / k)^{k}} \operatorname{value}\left(y^{\mathrm{lp}}, z^{\mathrm{lp}}\right) \text {. }
$$

The theorem is then proven.

To complete the proof of the theorem, we now proceed with the

Proof of Lemma 4.2. Consider $\left(y^{j}, z^{j}\right)$, for some $j \in[l: m]$, let us find an upper bound for value $\left(y^{j}, z^{j}\right)$. By construction:

$-z_{r}^{j}=1, \forall r \leq j$

$-z_{r}^{j}=0, \forall r \geq j+1$,

- $y^{j}$ is an optimal solution to $\operatorname{IP}^{y}(\mathcal{I}, b)$, where $b_{i}=1-z_{\rho_{i}}^{j}, \forall i \in[1: n]$.

Obviously, value $\left(z^{j}\right)=j$. In order to bound value $\left(y^{j}\right)$ we introduce a solution $y^{\prime j}$, which is feasible to the LP-relaxation of $\operatorname{IP}^{y}(\mathcal{I}, b)$. Then, Lemma 2.1 implies that value $\left(y^{j}\right) \leq \operatorname{value}\left(y^{\prime j}\right)$.

First, let us define subsets $S_{1}, S_{2}, \ldots, S_{m}$, where $S_{r} \subset[1: t], \forall r=1, \ldots, m$, (i.e., each subset consists of a set of columns of the grid) in the following way:

$$
S_{r}=\bigcup_{i: \rho_{i}=r}\left[l_{i}: r_{i}\right]
$$

Thus, $S_{r}$ is the set of columns stabbing intervals in row $r$. 
Fix now some $j \in[l: m]$, and construct $y^{\prime j}$ as follows (recall that $z_{r}^{\mathrm{p}}<$ $1, \forall r \in[l+1: m])$ :

$$
y_{c}^{\prime j}= \begin{cases}\frac{1}{\left(1-z_{j+1}^{\mathrm{lp}}\right)} y_{c}^{\mathrm{lp}}, & \text { if } c \in S_{j+1} \\ \frac{1}{\left(1-z_{j+2}^{\mathrm{lp}}\right)} y_{c}^{\mathrm{lp}}, & \text { if } c \in S_{j+2} \backslash S_{j+1} \\ \cdots & \\ \frac{1}{\left(1-z_{m}^{\mathrm{lp}}\right)} y_{c}^{\mathrm{lp}}, & \text { if } c \in S_{m} \backslash\left(S_{j+1} \cup \ldots \cup S_{m-1}\right) \\ y_{c}^{\mathrm{lp}} & \text { otherwise. }\end{cases}
$$

Let us now establish feasibility of $y^{\prime j}$ with respect to the LP-relaxation of $\operatorname{IP}^{y}(\mathcal{I}, b)$. For any interval $i$ we show that the following inequality holds:

$$
\sum_{c \in\left[l_{i}: r_{i}\right]} y_{c}^{\prime j} \geq 1-z_{\rho_{i}}^{j}
$$

If $\rho_{i}<j+1$, where $\rho_{i}$ is the row number of interval $i$, then $z_{\rho_{i}}^{j}=1$, and the inequality holds automatically. Consider the case $\rho_{i} \geq j+1$. Clearly, for any $c \in S_{\rho_{i}}$, either $c \in S_{\rho_{i}} \backslash\left(S_{j+1} \cup \ldots \cup S_{\rho_{i}-1}\right)$, or $c \in\left(S_{j+1} \cup \ldots \cup S_{\rho_{i}-1}\right)$. The first case implies that $y_{c}^{\prime j}=y_{c}^{\mathrm{lp}} /\left(1-z_{\rho_{i}}^{\mathrm{lp}}\right)$; the second means that $y_{c}^{\prime j}$ is equal to $y_{c}^{\mathrm{lp}}$ multiplied by some coefficient at least as large as $1 /\left(1-z_{\rho_{i}}^{\mathrm{lp}}\right)$ (see $(17)$ ). Then, since $\left[l_{i}: r_{i}\right] \in S_{\rho_{i}}$, we have $y_{c}^{\prime j} \geq y_{c}^{\mathrm{lp}} /\left(1-z_{\rho_{i}}^{\mathrm{lp}}\right)$ for any $c \in\left[l_{i}: r_{i}\right]$. Using this and remembering that $\left(y^{\mathrm{lp}}, z^{\mathrm{lp}}\right)$ satisfies $z_{\rho_{i}}^{\mathrm{lp}}+\sum_{c \in\left[l_{i}: r_{i}\right]} y_{c}^{\mathrm{lp}} \geq 1$, we have:

$$
\sum_{c \in\left[l_{i}: r_{i}\right]} y_{c}^{\prime j} \geq \frac{1}{\left(1-z_{\rho_{i}}^{\mathrm{lp}}\right)} \sum_{c \in\left[l_{i}: r_{i}\right]} y_{c}^{\mathrm{lp}} \geq \frac{1-z_{\rho_{i}}^{\mathrm{lp}}}{1-z_{\rho_{i}}^{\mathrm{p}}}=1 .
$$

Thus we have shown that inequality (18) holds for any $i \in[1: n]$, and therefore $y^{\prime j}$ is feasible to the LP-relaxation of $\operatorname{IP}^{y}(b)$. Now Lemma 2.1 implies that

$$
\operatorname{value}\left(y^{j}\right) \leq \operatorname{value}\left(y^{\prime j}\right)
$$

In what follows we show that value $\left(y^{\prime j}\right) \leq k \cdot q_{j}\left(z^{\text {lp }}, \frac{v a l u e\left(y^{\mathrm{lp}}\right)}{k}\right), \forall j \in[l: m]$. By construction of $y^{\prime j}$, using notation $Y(S)=\sum_{c \in S} y_{c}^{\text {lp }}$ :

$$
\begin{aligned}
& \operatorname{value}\left(y^{\prime j}\right)=\frac{1}{1-z_{j+1}^{\mathrm{lp}}} Y\left(S_{j+1}\right)+\frac{1}{1-z_{j+2}^{\mathrm{lp}}} Y\left(S_{j+2} \backslash S_{j+1}\right)+\ldots \\
& \ldots+\frac{1}{1-z_{m}^{\mathrm{lp}}} Y\left(S_{m} \backslash\left(S_{j+1} \cup S_{j+2} \cup \ldots \cup S_{m-1}\right)\right)+Y\left([1: t] \backslash\left(S_{j+1} \cup S_{j+2} \cup \ldots \cup S_{m}\right)\right) .
\end{aligned}
$$


Observe that for the $Y(\cdot)$-terms the following equality holds:

$$
\begin{aligned}
& Y\left(S_{j+1}\right)+Y\left(S_{j+2} \backslash S_{j+1}\right)+\ldots+Y\left(S_{m} \backslash\left(S_{j+1} \cup S_{j+2} \cup \ldots \cup S_{m-1}\right)\right)+ \\
& \quad+Y\left([1: t] \backslash\left(S_{j+1} \cup S_{j+2} \cup \ldots \cup S_{m}\right)\right)=\sum_{c=1}^{t} y_{c}^{\mathrm{lp}}=\operatorname{value}\left(y^{\mathrm{lp}}\right)
\end{aligned}
$$

Moreover, using the definition of $S_{r}$, our assumption (12), and the fact that there are at most $k$ intervals per row, we have for each $r=j+1, \ldots, m$ :

$$
\begin{aligned}
& Y\left(S_{r} \backslash\left(S_{j+1} \cup S_{j+2} \cup \ldots \cup S_{r-1}\right)\right) \leq Y\left(S_{r}\right)=\sum_{c \in S_{r}} y_{c}^{\mathrm{lp}}= \\
& \quad=\sum_{i: \rho_{i}=r} \sum_{c \in\left[l_{i}: r_{i}\right]} y_{c}^{\mathrm{lp}}=\sum_{i: \rho_{i}=r}\left(1-z_{\rho_{i}}^{\mathrm{lp}}\right) \leq k\left(1-z_{r}^{\mathrm{lp}}\right) .
\end{aligned}
$$

Consider now the following optimization problem:

$$
\begin{array}{cc}
\max _{Y_{j+1}, Y_{j+2}, \ldots}\left(\frac{1}{1-z_{j+1}^{\mathrm{lp}}} Y_{j+1}+\frac{1}{1-z_{j+2}^{\mathrm{lp}}} Y_{j+2}+\ldots+\frac{1}{1-z_{m}^{\mathrm{lp}}} Y_{m}+\sum_{r=m+1}^{\infty} Y_{r}\right) \\
\text { subject to } \\
Y_{j+1}+\ldots+Y_{m}+\sum_{r=m+1}^{\infty} Y_{r} \leq \operatorname{value}\left(y^{\mathrm{lp}}\right) \\
0 \leq Y_{r} \leq k\left(1-z_{r}^{\mathrm{lp}}\right), \quad \forall r=j+1, \ldots, m \\
0 \leq Y_{r} \leq k, \quad \forall r=m+1, \ldots, \infty
\end{array}
$$

Due to $(21)$ and (22) the following solution is feasible to it:

$Y_{r}=Y\left(S_{r} \backslash\left(S_{j+1} \cup S_{j+2} \cup \ldots \cup S_{r-1}\right)\right)$ for each $r=j+1, \ldots, m$, and $\sum_{r=m+1}^{\infty} Y_{r}=$ $Y\left((1: t) \backslash\left(S_{j+1} \cup S_{j+2} \cup \ldots \cup S_{m}\right)\right)$ (distributed arbitrary among the components of the sum). Therefore the optimum value of this optimization problem is an upper bound on the right-hand side of (20).

How does the optimum solution to this optimization problem look like? It is easy to see that to achieve the optimum value one should assign the maximum possible value (according to the constraints) to the variables with the largest objective coefficient. Since the objective coefficients are nonincreasing, we increase the values of the variables $Y_{j+1}, Y_{j+2}, \ldots$ in this order until the limits are met. We obtain the following optimal solution:

$$
\begin{gathered}
Y_{j+1}=k\left(1-z_{j+1}^{\mathrm{lp}}\right), Y_{j+2}=k\left(1-z_{j+2}^{\mathrm{p}}\right), \quad \ldots, \quad Y_{j+\lfloor q\rfloor}=k\left(1-z_{j+\lfloor q\rfloor}^{\mathrm{lp}}\right), \\
Y_{j+\lfloor q\rfloor+1}=(q-\lfloor q\rfloor) k\left(1-z_{j+\lfloor q\rfloor+1}^{\mathrm{lp}}\right),
\end{gathered}
$$

for some number $q \in \mathbb{R}_{+}$, which due to (23) has to satisfy:

$k\left(1-z_{j+1}^{\mathrm{lp}}\right)+k\left(1-z_{j+2}^{\mathrm{lp}}\right)+\ldots+k\left(1-z_{j+\lfloor q\rfloor}^{\mathrm{lp}}\right)+k(q-\lfloor q\rfloor)\left(1-z_{j+\lfloor q\rfloor+1}^{\mathrm{lp}}\right)=\operatorname{value}\left(y^{\mathrm{lp}}\right)$, 
where we put: $z_{r}^{\mathrm{lp}}=0$ for any $r>m$. Notice that $q \equiv q_{j}\left(z^{\mathrm{lp}}, \frac{\text { value }\left(y^{\mathrm{lp}}\right)}{k}\right)$ (see (14)), and the optimum value of the problem (23)-(25), which bounds the right-hand side of (20) from above, is $k \cdot q_{j}\left(z^{\mathrm{lp}}, \frac{\operatorname{value}\left(y^{\mathrm{lp}}\right)}{k}\right)$. This proves Lemma 4.2 .

Remark 4.1. With small adjustments, STAB and Theorem 4.1 can be generalized to the case of arbitrary rectangle demands. The main idea is then to sort the rows according to the fractional parts of the $z$-values of the optimal LP solution in case they are greater than 1.

Remark 4.2. It is proven in [5] that $S T A B$ also is a $e /(e-1)$-approximation algorithm for the weighted case of $\mathrm{ISP}_{\infty}$, i.e., the case where the columns and rows of the grid may have arbitrary integer positive weights. The proof given in this paper does not extend automatically to this case.

\subsection{Tightness.}

In this subsection we demonstrate that the ratio between the optimum values of $\operatorname{ISP}_{k}$ and the LP-relaxation of its ILP formulation (8)-(10) can be arbitrarily close to the bounds achieved by $S T A B$ in case $k=2$ and $k=\infty$ (which is respectively $4 / 3$ and $e /(e-1)$ ).

For the case $k=2$ this is shown by the instance of $\mathrm{ISP}_{2}$ depicted in Figure 5 (recall that all the column and row demands and rectangle weights are unit). Here the optimal value of the problem is 2 , since at least two elements (columns or rows) are needed to stab the 3 rectangles, whereas the optimal fractional solution has the value of $3 / 2$.

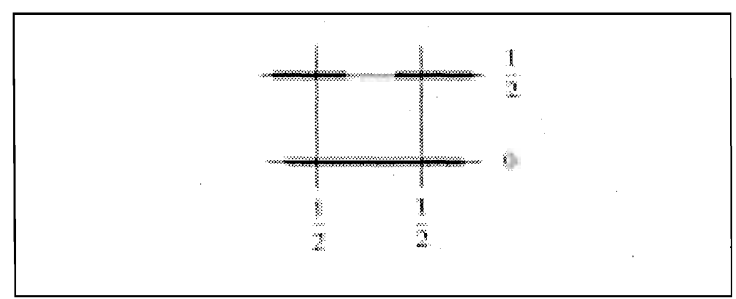

Figure 5: An instance of $\mathrm{ISP}_{2}$ and an optimal fractional solution.

In the remainder of the section we consider the problem ISP $_{\infty}$, or simply ISP, without any limitation on the number of rectangles sharing a row. We will exhibit a family of instances $\left\{\mathcal{I}_{m}\right\}_{m \in \mathbb{N}}$ of ISP, such that the ratio between the 
optimal values of integral and fractional solutions for $\mathcal{I}_{m}$ tends to $e /(e-1)$ as $m$ increases.

Theorem 4.4. The integrality gap of (8)-(10) is arbitrarily close to $\frac{e}{e-1}$.

Proof. For each $m \in \mathbb{N}$ we construct an instance $\mathcal{I}_{m}$ as follows. Let the grid have $m$ rows and $t=m$ ! columns. Let the rows be numbered consecutively and let each row $j$ intersect exactly $j$ rectangles of the instance. Let rectangles intersected by row $j$ be numbered $j_{1}, \ldots, j_{j}$. All these rectangles are disjoint and each intersects exactly $\frac{m !}{j}$ columns (see Figure 6). So, for a rectangle $j_{i}$ we have that its row number $\rho_{j_{i}}$ is $r$, and its leftmost and rightmost columns are $l_{j_{i}}=\frac{m !}{j}(i-1)+1$ and $r_{j_{i}}=\frac{m !}{j} i$. The total number of rectangles in the instance is then $n=1+2+\ldots+m$.

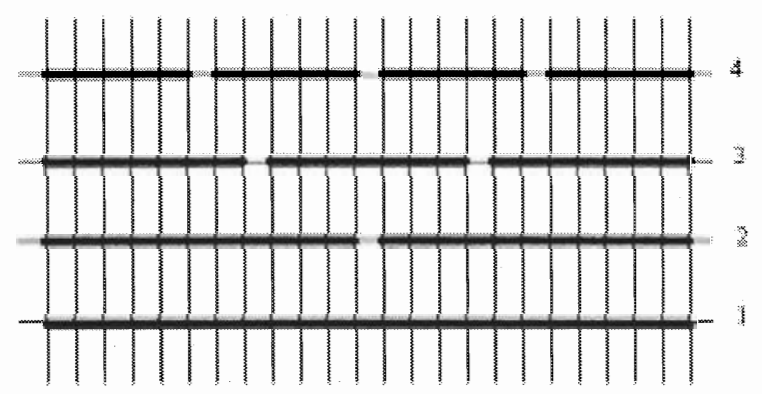

Figure 6: Instance $\mathcal{I}_{4}$.

We claim that the following solution $(y, z)$ is optimal to the LP-relaxation of (8)-(10) for $\mathcal{I}_{m}$ :

$$
\begin{aligned}
& z_{j}= \begin{cases}0, & \forall j=1, \ldots, P \\
1-P / j, & \forall j=P+1, \ldots, m\end{cases} \\
& y_{c}=\frac{P}{m !}, \forall c=1, \ldots, m !,
\end{aligned}
$$

where $P=P(m)$ is the number satisfying:

$$
\frac{1}{m}+\frac{1}{m-1}+\ldots+\frac{1}{P+1} \leq 1 \text { and } \frac{1}{m}+\frac{1}{m-1}+\ldots+\frac{1}{P+1}+\frac{1}{P} \geq 1 .
$$


It is easy to verify that the value of this solution equals:

$$
\sum_{c=1}^{t} y_{c}+\sum_{r=1}^{m} z_{r}=m-P\left(\frac{1}{P+1}+\frac{1}{P+2}+\ldots+\frac{1}{m}\right) .
$$

First, we show that $(y, z)$ is a feasible solution to the LP-relaxation. Take any rectangle $j_{i}$ and show that the constraint $z_{\rho_{j_{i}}}+\sum_{c \in\left[l_{j_{i}}, r_{j_{i}}\right]} y_{c} \geq 1$ is satisfied. Notice that the $z$-values of our solution can be also expressed as: $z_{j}=\max \left(1-\frac{P}{j}, 0\right), \forall j=1, \ldots, m$. Substituting these values, and rewriting the left-hand side of constraints (9) gives:

$$
\max \left(1-\frac{P}{j_{i}}, 0\right)+\sum_{c \in\left[l_{j_{i}}, r_{j_{i}}\right]} \frac{P}{m !}=\max \left(1-\frac{P}{j_{i}}, 0\right)+\frac{m !}{j_{i}} \frac{P}{m !}=\max \left(1-\frac{P}{j_{i}}, 0\right)+\frac{P}{j_{i}} .
$$

Clearly, the last expression is at least equal to 1 , which proves feasibility of the solution $(y, z)$.

Now, let us prove optimality of $(y, z)$. For this goal we present a feasible dual solution to the LP-relaxation of (8)-(10) which has the same value as $(y, z)$. Below we present the dual problem, where a variable $x_{i}$ corresponds to rectangle $i$ :

$$
\begin{array}{lcc}
\text { Maximize } & \sum_{i=1}^{n} x_{i} & \\
\text { subject to } & \sum_{i: \rho_{i}=j} x_{i} \leq 1 & \forall j=1, . ., m \\
& \sum_{i: c \in\left[l_{i}, r_{i}\right]} x_{i} \leq 1 & \forall c=1, . ., t \\
& 0 \leq x_{i} \leq 1 & \forall i=1, . ., n
\end{array}
$$

Consider now the following dual solution:

$$
x_{j_{i}}= \begin{cases}1 / j, & \text { if } j=P+1, \ldots, m \\ 1-\left(\frac{1}{m}+\frac{1}{m-1}+\ldots+\frac{1}{P+1}\right), & \text { if } j=P \\ 0 & \text { otherwise }\end{cases}
$$

In words, we assign $1 / j$ to the dual variables corresponding to rectangles on row $j=P+1, \ldots, m, 1-\left(\frac{1}{m}+\frac{1}{m-1}+\ldots+\frac{1}{P+1}\right)$ to the variables corresponding to rectangles on row $j=P$, and 0 to the other variables.

It is easy to verify that this solution is feasible to problem (27) and has value

$$
\sum_{i=1}^{n} x_{i}=m-P\left(\frac{1}{m}+\frac{1}{m-1}+\ldots+\frac{1}{P+1}\right)
$$


which is equal to the value of solution $(y, z)(26)$. This proves optimality of the latter to the LP-relaxation of (8)- (10). We denote the optimal value of this LP-relaxation by $L P\left(\mathcal{I}_{m}\right)$ (see $(29)$ ).

Denote by $O P T(\mathcal{I})$ the optimum value of ISP for $\mathcal{I}$. Let us show by induction on $m$ that $O P T\left(\mathcal{I}_{m}\right)=m$. It is clear that $O P T\left(\mathcal{I}_{1}\right)=1$, since exactly one element (column or row) is needed to stab the only rectangle of the instance $\mathcal{I}_{1}$. Assume now that $O P T\left(\mathcal{I}_{m-1}\right)=m-1$ is proven. Consider an instance $\mathcal{I}_{m}$. It is easy to see that $O P T\left(\mathcal{I}_{m}\right) \leq m$ (assign, for example, multiplicity 1 to each row). We claim that $O P T\left(\mathcal{I}_{m}\right) \geq m$. Indeed, take an optimal solution to ISP for $\mathcal{I}_{m},(y, z)$, and consider the following two cases. First, assume $z_{m}=0$. This implies that we have to stab all the $m$ non-overlapping rectangles on row $m$ with columns of the grid, which would require exactly $m$ columns and would imply $O P T\left(\mathcal{I}_{m}\right) \geq m$.

Second, assume $z_{m}=1$. In this case all rectangles intersecting row $m$ are stabbed by the row. Observe that the rectangles that are not yet stabbed can be considered separately and constitute instance $\mathcal{I}_{m-1}$. By the induction hypothesis we know that one has to select at least $m-1$ elements to stab all the rectangles in $\mathcal{I}_{m-1}$, and therefore $\operatorname{OPT}\left(\mathcal{I}_{m}\right) \geq 1+m-1=m$. So, $O P T\left(\mathcal{I}_{m}\right)$ has to be equal to $m$.

We use Lemma 6.3 given in the appendix to prove that the ratio

$$
\frac{O P T\left(\mathcal{I}_{m}\right)}{L P\left(\mathcal{I}_{m}\right)}=\frac{m}{m-P\left(\frac{1}{m}+\frac{1}{m-1}+\ldots+\frac{1}{P+1}\right)}
$$

approaches $\frac{e}{e-1}$ when $m$ increases. This establishes our tightness result.

As mentioned in the introduction, Theorems 4.1 and 4.4 imply that it is unlikely that a better ratio for $\mathrm{ISP}_{\infty}$ can be achieved using formulation (8)(10). Another example of a formulation with an integrality gap that equals $\frac{e}{e-1}$ is described in Hoogeveen et al. [4].

\section{Conclusion}

We discussed two variants of the weighted rectangle stabbing problem. For one problem, called WRSPq, an approximation algorithm ROUND is proposed that achieves a ratio of $\frac{q}{q-1}$. For the other problem, called $\mathrm{ISP}_{k}$, an approximation algorithm $S T A B$ is proposed that achieves a ratio of $\frac{1}{1-(1-1 / k)^{k}}$. 
Each of these algorithms is based on rounding the LP-relaxation of a straightforward integer programming formulation. ROUND is a generalization of an algorithm proposed in Gaur et al. [2], and it is shown that the ratio proved equals the integrality gap. $S T A B$ considers different ways of rounding the LP-relaxation, and outputs the best solution found in this way; again, it is shown that the ratio proved equals the integrality gap when $k=2$ and when $k=\infty$.

\section{Appendix.}

We restate Lemma 4.3, before giving its proof.

Lemma 4.3 Given are real numbers $1 \geq \Delta_{1} \geq \Delta_{2} \geq \ldots \geq \Delta_{m} \geq 0$, a positive real number $Y$, an integer $p \geq 2$ and an integer $0 \leq l<m$. The following holds:

$$
\min _{i=l, \ldots, m}\left(i+p \cdot q_{i}(\Delta, Y / p)\right) \leq \frac{1}{1-(1-1 / p)^{p}}\left(Y+\sum_{r=l+1}^{m} \Delta_{r}\right)+l,
$$

where $q_{i}=q_{i}(\Delta, Y / p)$ for each $i \in[0: m]$ is uniquely defined by the equality:

$$
\left(1-\Delta_{i+1}\right)+\left(1-\Delta_{i+2}\right)+\ldots+\left(1-\Delta_{i+\left\lfloor q_{i}\right\rfloor}\right)+\left(q_{i}-\left\lfloor q_{i}\right\rfloor\right)\left(1-\Delta_{i+\left\lfloor q_{i}\right\rfloor+1}\right)=Y / p
$$

where we put $\Delta_{i}=0$, if $i>m$.

Proof. It is enough to prove this lemma for $l=0$. The case of other $l<m$ can be reduced to the case of $l=0$ by changing the index to $j=i-l$ and observing that $q_{j+l}(\Delta, \alpha)=q_{i}\left(\Delta^{-l}, Y / p\right)$, where vector $\Delta^{-l}$ is obtained by deleting the first $l$ elements from vector $\Delta$. So we will prove that

$$
\min _{i=0, \ldots, m}\left(i+p \cdot q_{i}(\Delta, Y / p)\right) \leq \frac{1}{1-(1-1 / p)^{p}}\left(Y+\sum_{r=1}^{m} \Delta_{r}\right),
$$

The proof consists of two lemmas. In Lemma 6.1 we show that the lefthand side of (30) is upper bounded by the following supremum:

$$
\sup _{f(\cdot) \in H} G(f(\cdot))
$$


where

$$
G(f(\cdot))=\min _{x \in \mathbb{R}_{+}}(f(x)+k(f(x+Y / p)-f(x))),
$$

and the class of functions $H$ is defined as follows:

$$
H=\left\{\begin{array}{l|l}
f(\cdot): \mathbb{R}_{+} \rightarrow \mathbb{R}_{+} & \begin{array}{l}
f(\cdot) \text { is continuous, increasing, concave, } \\
f(0)=0, f(x) \leq x+\sum_{r=1}^{m} \Delta_{r}
\end{array}
\end{array}\right\} .
$$

In Lemma 6.2 we show, that this supremum is upper bounded by the righthand side of (30), which proves the lemma.

Lemma 6.1.

$$
\min _{i=0, \ldots, m}\left(i+p \cdot q_{i}(\Delta, Y / p)\right) \leq \sup _{f(\cdot) \in H} G(f(\cdot))
$$

where $G(f(\cdot))$ and $H$ are defined in (33) and (34).

Proof. To establish this, it is sufficient to exhibit a particular function $\hat{f}(\cdot) \in H$, such that:

$$
G(\hat{f}(\cdot))=\min _{i=0, \ldots, m}\left(i+p \cdot q_{i}(\Delta, Y / p)\right) .
$$

Then, the supremum of $G(f(\cdot))$ over all the possible $f(\cdot) \in H$ is clearly larger or equal to $G(\hat{f}(\cdot))$.

Before we describe the function $\hat{f}(\cdot)$, let us define an auxiliary function $F(\cdot)$ : $\mathbb{R}_{+} \rightarrow \mathbb{R}_{+}$as follows:

$$
F(q) \equiv \sum_{r=1}^{\lfloor q\rfloor}\left(1-\Delta_{r}\right)+(q-\lfloor q\rfloor)\left(1-\Delta_{\lfloor q\rfloor+1}\right)
$$

where we set $\Delta_{r}=0, \forall r \geq m+1$.

Observe that $F(\cdot)$ is

- continuous,

- increasing, since $\Delta_{r}<1$, and therefore $\left(1-\Delta_{r}\right)>0, \forall r=1, \ldots, \infty$,

- convex, since the coefficients $\Delta_{r}$ are non-increasing with increasing $r$, and therefore the coefficients $\left(1-\Delta_{r}\right)$ are non-decreasing with increasing $r$, 
$-F(0)=0$

- $F(q) \geq\left(q-\sum_{r=1}^{m} \Delta_{r}\right), \forall q \in \mathbb{R}_{+}$, since $F(q)$ can be also represented as:

$$
F(q)=q-\left(\sum_{r=1}^{\lfloor q\rfloor} \Delta_{r}+(q-\lfloor q\rfloor) \Delta_{\lfloor q\rfloor+1}\right),
$$

and obviously $\left(\sum_{r=1}^{\lfloor q\rfloor} \Delta_{r}+(q-\lfloor q\rfloor) \Delta_{\lfloor q\rfloor+1}\right) \leq \sum_{r=1}^{m} \Delta_{r}, \forall q \in \mathbb{R}_{+}$,

- $F(q)$ is linear on each of the intervals $[i, i+1], i=0, \ldots, m-1$, and on $[m,+\infty)$.

We are now ready to present $\hat{f}(\cdot): \mathbb{R}_{+} \rightarrow \mathbb{R}_{+}$. We define:

$$
\hat{f}(\cdot) \equiv F^{-1}(\cdot)
$$

(since $F(\cdot)$ is increasing, $F^{-1}(\cdot)$ exists.)

We claim that $\hat{f}(\cdot) \in H$. Indeed, $\hat{f}(\cdot)$ has the following properties:

- $\hat{f}(\cdot): \mathbb{R}_{+} \rightarrow \mathbb{R}_{+}$since $F(\cdot): \mathbb{R}_{+} \rightarrow \mathbb{R}_{+}$;

- $\hat{f}(\cdot)$ is continuous, increasing, concave, since $F(\cdot)$ is continuous, increasing, convex;

- $\hat{f}(0)=0$, since $F(0)=0$;

- $\hat{f}(x) \leq x+\sum_{r=1}^{m} \Delta_{r}, \forall x \in \mathbb{R}_{+}$. This can be obtained from $F(q) \geq(q-$ $\left.\sum_{r=1}^{m} \Delta_{r}\right), \forall q \in \mathbb{R}_{+}$, using: $F(q)=x, q=\hat{f}(x)$.

This proves that $\hat{f}(\cdot) \in H$.

To prove the lemma it remains to show that

$$
G(\hat{f}(\cdot))=\min _{i=0, \ldots, m}\left(i+p q_{i}(\Delta, Y / p)\right) .
$$

Comparing the definition of $q_{i}(\Delta, Y / p)$ (see 31) and $F(\cdot)$ (36), observe that for each $i \in[0: m] q_{i}$ satisfies:

$$
F\left(i+q_{i}\right)-F(i)=Y / p .
$$

Thus, $q_{i}=F^{-1}(F(i)+Y / p)-i$. Setting: $x_{i} \equiv F(i), \forall i=0, \ldots, m$, we find that $i=F^{-1}\left(x_{i}\right)$ and $\left.q_{i}=F^{-1}\left(x_{i}+Y / p\right)\right)-F^{-1}\left(x_{i}\right)$. Replacing $F^{-1}(\cdot)$ by $\hat{f}(\cdot)$, we obtain:

$$
\left.q_{i}=\hat{f}\left(x_{i}+Y / p\right)\right)-\hat{f}\left(x_{i}\right), \forall i=0, \ldots, m .
$$


Using this together with $i=F^{-1}\left(x_{i}\right)=\hat{f}\left(x_{i}\right)$, we can rewrite:

$$
\begin{aligned}
\min _{i=0, \ldots, m}\left(i+p q_{i}(\Delta, Y / p)\right)= & \min _{i=0, \ldots, m}\left(\hat{f}\left(x_{i}\right)+p\left(\hat{f}\left(x_{i}+Y / p\right)-\hat{f}\left(x_{i}\right)\right)\right) \\
& x_{i}=\hat{f}^{-1}(i)
\end{aligned}
$$

Now we need to show that the latter expression is equal to:

$$
G(\hat{f}(\cdot)) \equiv \min _{x \in \mathbb{R}_{+}}(\hat{f}(x)+p(\hat{f}(x+Y / p)-\hat{f}(x)))
$$

We do this by showing that the function $\hat{f}(x)+p(\hat{f}(x+Y / p)-\hat{f}(x))$ is continuous and concave in each of the intervals $\left[x_{i}, x_{i+1}\right], \forall i=0, \ldots, m-1$, and is increasing in $\left[x_{m},+\infty\right)$. Therefore the minimum can be achieved only at one of the endpoints $x_{0}, x_{1}, \ldots, x_{m}$.

Indeed, consider function $\hat{f}(x)+p(\hat{f}(x+Y / p)-\hat{f}(x))$ in $\left[x_{i}, x_{i+1}\right]$ for some $i \in[0: m-1]$. It can also be written as $p \hat{f}(x+Y / p)-(p-1) \hat{f}(x)$. We know that $\hat{f}(x+Y / p)$ is concave on $\left[x_{i}, x_{i+1}\right]$, since it is concave everywhere in $\mathbb{R}_{+}$. Furthermore, $\hat{f}(x)$ is linear on each $\left[x_{i}, x_{i+1}\right], i \in[0: m-1]$, since $F(\cdot)$ is linear on $[i, i+1], i \in[0: m-1]$. Obviously, a concave function minus a linear function is again concave.

Now we show that $p \hat{f}(x+Y / p)-(p-1) \hat{f}(x)$ is increasing in $\left[x_{m},+\infty\right)$. Since $\hat{f}(x)=F^{-1}(\cdot)$ is increasing and linear in $\left[x_{m},+\infty\right)$, the growth rate of $\hat{f}(x)$ is the same as the growth rate of $\hat{f}(x+Y / p)$ in $\left[x_{m},+\infty\right)$, and thus the growth rate of $p \hat{f}(x+Y / p)-(p-1) \hat{f}(x)$ is negative. We have proved that the minimum in (39) is always achieved at one of the points $x_{0}, x_{1}, \ldots, x_{m}$, and therefore (39) is equal to (38). This completes the proof of Lemma 6.1.

\section{Lemma 6.2.}

$$
\sup _{f(\cdot) \in H} G(f(\cdot)) \leq \frac{1}{1-(1-1 / k)^{k}} C
$$

where

$$
\begin{gathered}
C=Y+\sum_{r=1}^{m} \Delta_{r}, \\
G(f(\cdot))=\min _{x \in \mathbb{R}_{+}}(f(x)+k(f(x+Y / p)-f(x)))
\end{gathered}
$$

and set of functions $H$ (via notation $C$ ) is

$$
H=\left\{\begin{array}{l|l}
f(\cdot): \mathbb{R}_{+} \rightarrow \mathbb{R}_{+} & \begin{array}{l}
f(\cdot) \text { is continuous, increasing, concave, } \\
f(0)=0, f(x) \leq x+C-Y
\end{array}
\end{array}\right\} .
$$


Proof. We will prove several claims and sub-claims.

\section{Claim 1.}

$$
\sup _{f(\cdot) \in H} G(f(\cdot))=\sup _{g: f^{g}(\cdot) \in H} g
$$

where for each $g \in \mathbb{R}_{+}$function $f^{g}(\cdot)$ is defined as follows:

- $f^{g}(j \cdot Y / p)=g\left(1-(1-1 / p)^{j}\right), \forall j \in 0 \cup \mathbb{N}$,

- $f^{g}(x)$ is continuous in $[0,+\infty)$ and linear in each $[(j-1) \cdot Y / p, j \cdot Y / p], j \in \mathbb{N}$.

Notice that $f^{g}(\cdot)$ is completely defined by the above characterization. An example of this function is shown in Figure 7.

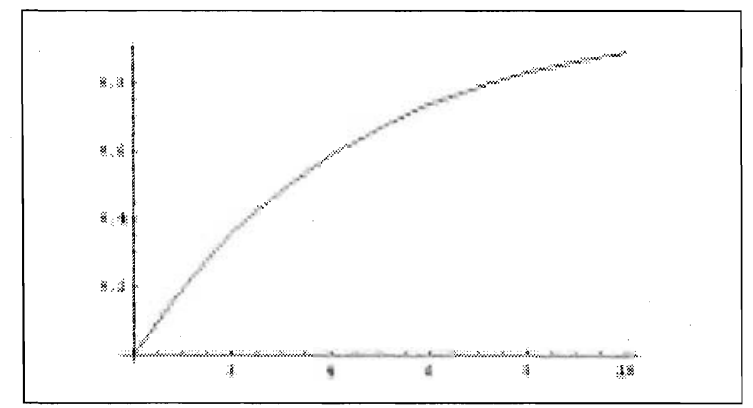

Figure 7: Illustration of function $f^{g}(x)$ for $k=5, g=1$.

To prove this claim it is enough to show that for any $f(\cdot) \in H$ there exists a function $f^{\hat{g}}(\cdot) \in H$, with $\hat{g} \geq 0$, such that

$$
G(f(\cdot))=G\left(f^{\hat{g}}(\cdot)\right)=\hat{g} .
$$

To show that, we prove 2 subsidiary claims.

Claim 1.1. For any $g \geq 0$ :

$$
G\left(f^{g}(\cdot)\right) \equiv \min _{x \in \mathbb{R}_{+}}\left(f^{g}(x)+p\left(f^{g}(x+Y / p)-f^{g}(x)\right)\right)=g .
$$

Indeed, by construction $f^{g}(x)$ is linear in each of the intervals $[(j-1) \cdot Y / p, j$. $Y / p], j \in \mathbb{N}$. This implies that function $\left(f^{g}(x)+p\left(f^{g}(x+Y / p)-f^{g}(x)\right)\right)$ is linear in each of these intervals as well. Therefore the minimum over all $x \geq 0$ is achieved in one of the endpoints $0, Y / p, 2 Y / p, \ldots$. Consider $\left(f^{g}(x)+p\left(f^{g}(x+Y / p)-f^{g}(x)\right)\right)$ at the point $x=j Y / p$, for some $j \in \mathbb{N} \cup 0$ :

$$
f^{g}(j \cdot Y / p)+p\left(f^{g}((j+1) \cdot Y / p)-f^{g}(j \cdot Y / p)\right) .
$$


Using the definition of $f^{g}(\cdot)$ we can rewrite it as follows:

$$
g\left(1-(1-1 / p)^{j}\right)+p\left(g\left(1-(1-1 / p)^{j+1}\right)-g\left(1-(1-1 / p)^{j}\right)\right)
$$

With simple computations one can verify that the last expression is equal to g. This proves Claim 1.1.

Claim 1.2. For any $f(\cdot) \in H$ it holds that $f^{\hat{g}}(\cdot) \in H$, where $\hat{g}=G(f(\cdot))$. Clearly, $f^{\hat{g}}(x)$ is concave. To prove that $f^{\hat{g}}(x) \leq x+C-Y, \forall x \in \mathbb{R}_{+}$, it is sufficient to show that $f^{\hat{g}}(x) \leq f(x)$, since $f(\cdot) \in H$ means e.g. $f(x) \leq$ $x+C-Y, \forall x \in \mathbb{R}_{+}$.

So, let us establish that $f^{\hat{g}}(x) \leq f(x), \forall x \in \mathbb{R}_{+}$. Recall that $f^{\hat{g}}(x)$ is linear in each of the intervals $[(j-1) \cdot Y / p, j \cdot Y / p], j \in \mathbb{N}$, and $f(x)$ is concave in $\mathbb{R}_{+}$. Then it is sufficient to show that:

$$
f^{\hat{g}}(x) \leq f(x), \forall x=j \cdot Y / p, j \in 0 \cup \mathbb{N} .
$$

We use mathematical induction on $j$. For $j=0, f^{\hat{g}}(0)=f(0)=0$ and the inequality trivially holds. Suppose, for $j-1$ we have proven: $f^{\hat{g}}((j-1)$. $Y / p) \leq f((j-1) \cdot Y / p)$, and let us show that $f^{\hat{g}}(j \cdot Y / p) \leq f(j \cdot Y / p)$.

Observe, that $f^{\hat{g}}(\cdot)$ can be represented in a recursive way as follows:

$$
f^{\hat{g}}(j \cdot Y / p)=\hat{g} / p+f^{\hat{g}}((j-1) \cdot Y / p)(1-1 / p) .
$$

Since $\hat{g}=G(f(\cdot))$ we know:

$$
\hat{g} \leq f((j-1) \cdot Y / p)+p(f(j \cdot Y / p)-f((j-1) \cdot Y / p)) .
$$

Rearranging the expression, we obtain:

$$
f(j \cdot Y / p) \geq \hat{g} / p+f((j-1) \cdot Y / p)(1-1 / p) .
$$

By the induction hypothesis and (40) we can bound the right-hand side as: $\hat{g} / p+f((j-1) \cdot Y / p)(1-1 / p) \geq \hat{g} / p+f^{\hat{g}}((j-1) \cdot Y / p)(1-1 / p)=f^{\hat{g}}(j \cdot Y / p)$.

This proves Claim 1.2.

These 2 claims imply that for any $f(\cdot) \in H$, there exists $f^{\hat{g}}(\cdot) \in H$, with $\hat{g} \geq 0$, such that

$$
G(f(\cdot))=G\left(f^{\hat{g}}(\cdot)\right)=\hat{g} .
$$


This implies Claim 1.

\section{Claim 2.}

$$
\sup _{g: f^{g}(\cdot) \in H} g \leq \frac{1}{1-(1-1 / p)^{p}} C .
$$

Indeed, $f^{g}(\cdot) \in H$ implies: $f^{g}(x) \leq x+C-Y$, for all $x \in \mathbb{R}_{+}$, and in particular, for $x=Y$. From this, using the definition of $f^{g}(\cdot)$, obtain:

$$
f^{g}(Y) \equiv f^{g}(p * Y / p) \equiv g\left(1-(1-1 / p)^{p}\right) \leq Y+C-Y=C,
$$

from the last inequality:

$$
g \leq \frac{1}{\left(1-(1-1 / p)^{p}\right)} C,
$$

which proves Claim 2 and establishes Lemma 6.2.

Lemma 6.3. Let $P(m) \in \mathbb{N}$ be defined as follows:

$$
\begin{gathered}
\frac{1}{m}+\frac{1}{m-1}+\ldots+\frac{1}{P(m)+1} \leq 1 \text { and } \\
\frac{1}{m}+\frac{1}{m-1}+\ldots+\frac{1}{P(m)+1}+\frac{1}{P(m)} \geq 1 .
\end{gathered}
$$

Then,

$$
\lim _{m \rightarrow \infty} \frac{m}{m-P(m)\left(\frac{1}{m}+\frac{1}{m-1}+\ldots+\frac{1}{P(m)+1}\right)}=\frac{e}{e-1} .
$$

Proof. Let us first find $\lim _{m \rightarrow \infty} P(m) / m$. Observe that the following inequalities hold:

$$
\begin{gathered}
\frac{1}{m}+\frac{1}{m-1}+\ldots+\frac{1}{P(m)+1} \geq \int_{P(m)+1}^{m+1} \frac{1}{x} d x=\ln \frac{m+1}{P(m)+1}, \\
\frac{1}{m}+\frac{1}{m-1}+\ldots+\frac{1}{P(m)} \leq \int_{P(m)-1}^{m} \frac{1}{x} d x=\ln \frac{m}{P(m)-1},
\end{gathered}
$$

(the equalities follow from $\int_{a}^{b} 1 / x d x=\ln b / a$.) Then (41) and (42) imply

$$
1 \geq \ln \frac{m+1}{P(m)+1}, \quad 1 \leq \ln \frac{m}{P(m)-1} .
$$


From this we have:

$$
\frac{m+1}{e}-1 \leq P(m) \leq \frac{m}{e}+1
$$

Dividing by $m$ :

$$
\frac{1+1 / m}{e}-1 / m \leq \frac{P(m)}{m} \leq \frac{1}{e}+1 / m
$$

Now we see, that $\lim _{m \rightarrow \infty} P(m) / m=1 / e$.

Let us now find $\lim _{m \rightarrow \infty}\left(\frac{1}{m}+\frac{1}{m-1}+\ldots+\frac{1}{P(m)+1}\right)$. From (41) and (42) we have:

$$
1-\frac{1}{P(m)} \leq \frac{1}{m}+\frac{1}{m-1}+\ldots+\frac{1}{P(m)+1} \leq 1
$$

Since we already know that $\lim _{m \rightarrow \infty} P(m)=\infty$, we have:

$$
\lim _{m \rightarrow \infty}\left(\frac{1}{m}+\frac{1}{m-1}+\ldots+\frac{1}{P(m)+1}\right)=1
$$

Now consider:

$$
\frac{m}{m-P(m)\left(\frac{1}{m}+\frac{1}{m-1}+\ldots+\frac{1}{P(m)+1}\right)}=\frac{1}{1-\frac{P(m)}{m}\left(\frac{1}{m}+\frac{1}{m-1}+\ldots+\frac{1}{P(m)+1}\right)} .
$$

Using $\lim _{m \rightarrow \infty} \frac{P(m)}{m}=1 / e$ and $\lim _{m \rightarrow \infty}\left(\frac{1}{m}+\frac{1}{m-1}+\ldots+\frac{1}{P(m)+1}\right)=1$ we have:

$$
\lim _{m \rightarrow \infty} \frac{1}{1-\frac{P(m)}{m}\left(\frac{1}{m}+\frac{1}{m-1}+\ldots+\frac{1}{P(m)+1}\right)}=\frac{1}{1-1 / e}=\frac{e}{e-1}
$$

which establishes the lemma.

\section{References}

[1] Ahuja, R.K., T.L. Magnanti, and J.B. Orlin [1993], Network Flows: Theory, Algorithms, and Applications, Prentice-Hall. 
[2] Gaur, D.R., T. Ibaraki, and R. Krishnamurti [2002], Constant ratio approximation algorithms for the rectangle stabbing problem and the rectilinear partitioning problem, Journal of Algorithms 43, 138-152.

[3] Hassin, R., and N. Megiddo [1991], Approximation algorithm for hitting objects with straight lines, Discrete Applied Mathematics, 30, 29-42.

[4] Hoogeveen H., M. Skutella, and G.J. Woeginger [2003], Preemptive scheduling with rejection, Mathematical Programming 94, 361-374.

[5] Kovaleva, S. [2003], Approximation of Geometric Set Packing and Hitting Set Problems, Ph.D. thesis of Maastricht University, Maastricht, The Netherlands.

[6] Kovaleva, S., and F.C.R. Spieksma (2001), Approximation of a geometric set covering problem, in: Proceedings of the 12th Annual International Symposium on Algorithms and Computation (ISAAC'01), Lecture Notes in Computer Science 2223, 493-501.

[7] Kovaleva, S., and F.C.R. Spieksma [2002], Primal-dual approximation algorithms for a packing-covering pair of problems, RAIRO-Operations Research 36, 53-72.

[8] Schrijver, A. [1986], Theory of Linear and Integer Programming, Wiley.

[9] Vazirani, V.V. [2003], Approximation Algorithms, Springer. 

